\title{
Tanshinone IIA Attenuates Contrast- Induced Nephropathy via Nrf2 Activation in Rats
}

\author{
Rulian Liang ${ }^{\mathrm{a}}$ Qing Zhao ${ }^{\mathrm{b}} \quad$ Guihua Jian $^{\mathrm{a}}$ Dongsheng Cheng ${ }^{\mathrm{a}}$ Niansong Wang ${ }^{\mathrm{a}}$ \\ Guangyuan Zhangc Feng Wang ${ }^{c}$ \\ aDepartment of Nephrology, Shanghai Jiao Tong University Affiliated Sixth People's Hospital, Shanghai, \\ 'Department of Cardiology, Shanghai Jiao Tong University Affiliated Sixth People's Hospital, Shanghai, \\ 'Department of Nephrology, Shanghai Jiao Tong University Affiliated Sixth People's Hospital, Shanghai \\ Eighth People's Hospital, Shanghai, China
}

\section{Key Words}

Tanshinone IIA • Contrast-induced nephropathy • Oxidative stress • Nrf2

\begin{abstract}
Background/Aims: Tanshinone IIA is a chemical compound extracted from Salvia miltiorrhiza Bunge, a perennial plant also known as red sage used in traditional Chinese medicine. Tanshinone IIA has been shown to protect against various organ injuries. In this study, we hypothesized that Tanshinone IIA could play an anti-oxidative role in contrast-induced nephropathy (CIN) through enhancing Nrf2/ARE activation. Methods: To test whether Tanshinone IIA can attenuate CIN, oxidative stress, and apoptosis, we utilized two models: an in vivo Sprague-Dawley rat model of ioversol-induced CIN and an in vitro cell model of oxidative stress in which HK2 cells, a human renal tubular cell line, are treated with hydrogen peroxide $\left(\mathrm{H}_{2} \mathrm{O}_{2}\right)$. Rats were randomly assigned to 4 groups ( $n=6$ per group): control group, ioversol group (ioversol-induced CIN), vehicle group (ioversol-induced CIN rats pretreated with vehicle), and Tanshinone IIA group (ioversol-induced CIN rats pretreated with $25 \mathrm{mg} / \mathrm{kg}$ Tanshinone IIA). Renal functions, renal injuries and apoptosis were evaluated by using serum creatinine, histological scoring, and TUNEL staning respectively. Malondialdehyde, 8-hydroxy-2' -deoxyguanosine, and intracellular reactive oxygen species were used for oxidative stress assessment. Levels of Nrf2 and heme oxygenase-1 (HO-1) were measured in vivo and in vitro. Results: Tanshinone IIA attenuated renal tubular necrosis, apoptosis and oxidative stress in rats and oxidative stress in HK2 cells. Furthermore, Tanshinone IIA activated Nrf2, and up-regulated HO-1 expression in vivo and in vitro, resulting in a reduction in oxidative stress. Conclusion: Tanshinone IIA may protect against CIN through enhancing Nrf2/ARE activation.
\end{abstract}

L. Liang, Q. Zhao and G. Jian contributed equally to this work 
Liang et al.: Tanshinone Protects Against CIN

\section{Introduction}

The use of contrast media is becoming more and more common in radiology diagnosis [1]. Contrast-induced nephropathy (CIN) is a frequent complication. The incidence of CIN varies from $3 \%$ to $14 \%$ and can reach as high as $20 \%$ in patients with high risk $[2,3]$. Recent evidence suggests that CIN can cause long-term decline in renal function, leading to increased mortality $[4,5]$. These detrimental effects demonstrate the necessity for the development of new preventive strategies for CIN.

A variety of molecules contained in the roots of the perennial plant Salvia miltiorrhiza Bunge can be extracted [6, 7]. Some of these molecules have been widely applied in traditional Chinese medicine, especially in the treatment of cardiovascular and cerebrovascular diseases. Tanshinone IIA is an active monomer extracted from S. miltiorrhiza [8], which plays a key role in the plant's clinical efficacy. Previous studies suggest that Tanshinone IIA is neuroprotective in cerebral ischemia reperfusion injury $[8,9]$ and renoprotective in renal ischemia reperfusion injury [10].. However, the effects of Tanshinone IIA in CIN have not been investigated.

The mechanism of action for Tanshinone IIA remains unknown. Previous studies suggest that Tanshinone IIA may regulate proteins involved in apoptosis or necrosis, including B-cell lymphoma-2 (Bcl-2), Bcl-2-associated X protein (Bax), Transient receptor potential cation channel subfamily M member 7 (TRPM7), and the PI3K/AKT signaling pathway [11]. Additionally, Tanshinone IIA has been shown to have anti-oxidative properties in hepatic cell injury [12].

Nuclear factor erythroid 2 (Nrf2) is a pleiotropic transcription factor that plays a crucial role in maintaining homeostasis during intracellular and extracellular stress, by helping cells adapt to detrimental conditions [13]. Once Nrf2 is activated, it enters the nucleus and binds to antioxidant response elements (AREs), inducing transcription of a large number of antioxidant genes including heme oxygenase-1 (HO-1) [14]. A recent study showed that Nrf2 activation contributes to the neuroprotective effects of Tanshinone IIA during ischemic stroke through its anti-oxidative properties [15]. In the present study, we observed the effects of Tanshinone IIA preteatment on CIN induction, and investigated the underlying mechanism, which we proposed occurred through the Nrf2/ARE pathway.

\section{Materials and Methods}

\section{Reagents and Animals}

The Tanshinone IIA reagents were provided by Jiangsu Carefree Group Co. (Nanjing, China). Tanshinone IIA was solubilized in saline with $0.02 \%$ DMSO for administration. Male Sprague-Dawley rats from Shanghai Science Academy animal center, weighing $200 \pm 20 \mathrm{~g}$, were housed in individual cages under controlled conditions for light $\left(12 \mathrm{~h}\right.$ dark $/ 12 \mathrm{~h}$ light cycle) and temperature $\left(20-23^{\circ} \mathrm{C}\right)$. All rats were provided standard diet and tap water ad libitum.

\section{Animal study and group assign}

The animal experiments were approved by the Animal Care and Ethics Committee of Shanghai Jiao Tong University Affiliated Sixth People's Hospital. The CIN rat model was conducted as previously described [16]. The rats were divided into control group (Ctl) $(n=6)$, ioversol group (Iov) $(n=6)$, vehicle group (Veh) $(n=6)$, and Tanshinone IIA group (Tan) $(n=6)$. Rats in the Iov, Veh, and Tan groups were anesthetized with $50 \mathrm{mg} / \mathrm{kg}$ pentobarbital and given a tail vein injection of indomethacin (Sigma, USA) (10mg/kg), followed by ioversol (Hengrui Corp., China) (3g/kg organically bound iodine). Rats in the Ctl group received injections of saline alone at each time point. Rats in the Tan group received a subcutaneous injection of Tanshinone IIA $(25 \mathrm{mg} / \mathrm{kg}) 15$ minutes prior to the CIN inducing injections. Rats in the Veh group received a subcutaneous injection of vehicle (saline with $0.02 \%$ DMSO) $(25 \mathrm{mg} / \mathrm{kg}) 15$ minutes prior to the CIN inducing injections. The rats recovered in metabolic cages for 24 hours for urine collection. After 24 hours, the left kidneys 


\section{Cellular Physiology Cell Physiol Biochem 2018;46:2616-2623

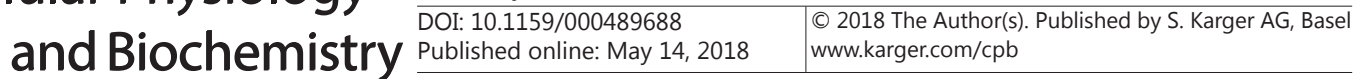

Liang et al.: Tanshinone Protects Against CIN

were harvested for associated measurements. The right kidneys were fixed in $10 \%$ formalin for histological assessments. Blood was collected to isolate serum and then stored in a $-80^{\circ} \mathrm{C}$ freezer.

\section{Renal function}

Serum creatinine was measured using a colorimetric microplate assay based on the Jaffe reaction (BioAssay Systems, Hayward, CA, USA). A colorimetric assay kit was used to quantify the levels of blood urea nitrogen (BUN) in accordance with the manufacturer's protocol (BioAssay Systems).

\section{Histological examinations}

Right kidneys were fixed in $10 \%$ formalin, then dehydrated in ethanol and embedded in paraffin. Kidney tissue blocks were cut into $4 \mu \mathrm{m}$ sections and subjected to Periodic Acid Schiff (PAS) staining. The sections were viewed by light microscopy. The histological scoring was assessed by the following criteria for the presence of acute necrosis in proximal tubular cells: 0, none; 1, 0-10\%; 2, 11-25\%; 3, 26-45\%; 4, 46-75\% and 5, 76-100\%, as described previously [17]. For each animal, three renal tissue sections were selected and 10 random microscopic fields at 400X magnification in each slide were selected for scoring. Two independent pathologists were blinded to the experimental conditions, and evaluated the presence of acute necrosis in proximal tubular cells according to the scoring criteria. Renal cell apoptosis was assessed using TUNEL staining (Roche Diagnostics, Mannheim, Germany) as described previously [18].

\section{Examination of Lipid Peroxide}

Malondialdehyde (MDA), a product of reduction in the lipid peroxidation processes [19], was detected using a commercial kit (Nanjing Jiancheng, Jiangsu, China) for supernatant of the renal cortical homogenate, according to the manufacturer's instruction.

\section{ELISA assay}

Serum neutrophil gelatinase-associated lipocalin (sNAGL) and urinary kidney injury molecule-1 (uKIM-1) were measured using commercial ELISA kits (R\&D, USA). The concentration of 8-hydroxy-2' -deoxyguanosine (8-OHdG) was also assessed by commercial ELISA kits (Japan Institute for the Control of Aging, Shizuoka, Japan).

\section{ROS assay for HK2}

HK2 cells (ATCC, Manassas, USA) were cultured in K-SFM at $37^{\circ} \mathrm{C} 5 \% \mathrm{CO} 2$, supplemented with $5 \mathrm{ng} /$ $\mathrm{mL}$ human recombinant Epidermal growth factor (EGF) and $0.05 \mathrm{mg} / \mathrm{mL}$ bovine pituitary extract. HK2 cells were preconditioned with Tanshinone IIA $(40 \mu \mathrm{g} / \mathrm{mL})$ for $1 \mathrm{~h}$ at $37^{\circ} \mathrm{C}$. Then the cells were washed 3 times by PBS followed by $500 \mu \mathrm{mol} / \mathrm{L} \mathrm{H}_{2} \mathrm{O}_{2}$ incubation for $5 \mathrm{~min}$ at room temperature to induce oxidative stress injury. Intracellular reactive oxygen species (ROS) was measured with commercial kits (Cell Biolabs, San Diego, USA) using Dichloro-dihydro-fluorescein diacetate (DCFH-DA) method. Briefly, cells were harvested and washed three times with PBS, and stained with $10 \mathrm{mM}$ DCFH-DA in serum-free cell culture medium at $37^{\circ} \mathrm{C}$ for $30 \mathrm{~min}$, with inversion every $5 \mathrm{~min}$. The cells were then washed three times with serum-free medium, and the fluorescent product DCF was measured using a spectrofluorimeter with excitation at 484 $\mathrm{nm}$ and emission at $530 \mathrm{~nm}[20]$.

\section{Western blot}

Western blot was conducted following previously described methods [5]. The primary antibodies were rabbit antibodies to Nrf2 (dilution 1:200, Santa Cruz, USA) or HO-1 (dilution 1:1000, Santa Cruz, USA). Antibodies to histone H3 (dilution 1:500, Cell Signaling Technology, USA) or GAPDH (dilution 1:500, Santa Cruz, USA) were used as internal controls for the nuclear and cytosolic target proteins, respectively. Each membrane was incubated for 1-2 hours with a secondary antibody conjugated by peroxidase (1:200, Zhongshanjinqiao Biotech., China) at room temperature, detected by ECL regent (Millipore, US).

\section{HO-1 activity assay and ARE reporter assay}

HO-1 activity was determined with a commercial assay kit (Nanjing Jiancheng, Jiangsu, China). ARE reporter assay was used to confirm the transcriptional activity of Nrf2 on HK2 using a SignalTM ARE Reporter Assay Kit (SABiosciences, USA). The assay was carried out according to the manufacturer's instruction. 
Fig. 1. Tanshinone IIA alleviated histological change and decreased Scr and BUN A, PAS staining for kidney tissues (400X) and tubular scoring; B, serum creatinine; C, BUN. $* P<0.05$, versus Ctl group. \#P< 0.05 , versus Veh group.
A
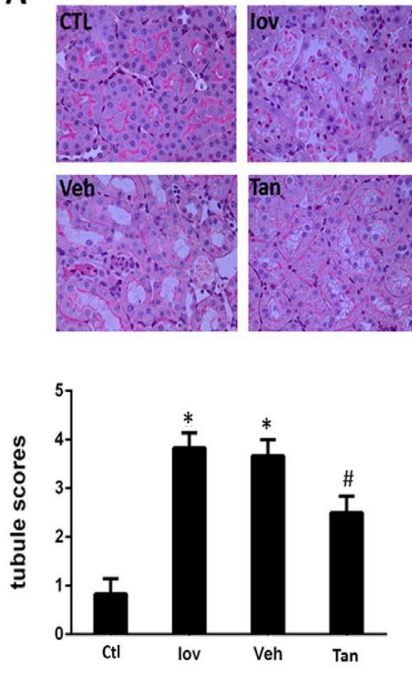

B

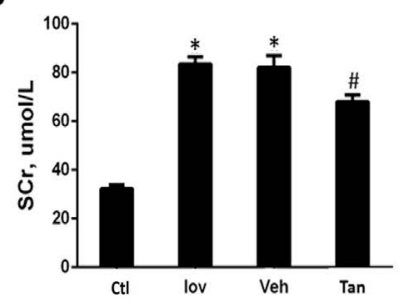

C

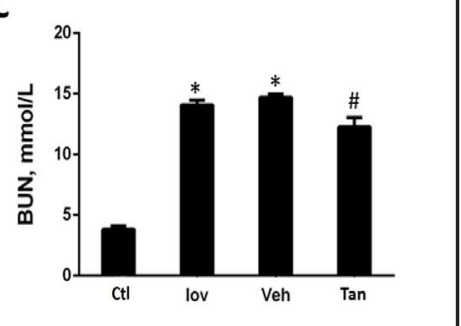

Statistical analysis

All the data were expressed as mean \pm SE. Statistical analysis was performed using SPSS software (Ver. 18.0, Chicago, IL, USA). Statistical significance was assessed by one-way analysis of variance (ANOVA). A value of $\mathrm{P}<0.05$ was considered to be statistically significant.

\section{Results}

Tanshinone IIA pretreatment alleviated renal injury

To evaluate the severity of tubular injury, PAS staining was performed. Tubular detachment, foamy degeneration, and necrosis were seen in kidney sections from rats with ioversol-induced CIN treatment (Fig. 1A). The morphological damage and tubular scores in Veh and Iov groups were much more severe than the Tan group. Serum creatinine and blood urea nitrogen also increased significantly in rats with ioversol-induced CIN treatment. However, serum creatinine and blood urea nitrogen in the rats of Tan group were significantly lower than the Veh and Iov groups (Fig. 1B and 1C).

\section{Tanshinone IIA mitigated renal apoptosis}

The TUNEL assay showed that apoptotic cell number increased with ioversol-induced CIN treatment, but the effect was significantly attenuated in the Tan group (Fig. 2A). To evaluated kidney cell injury, sNGAL and uKIM-1 were measured with ELISA assay [21]. In CIN rats, both uKIM-1 and sNGAL levels increased significantly while they were reduced in the Tan group (Fig. 2B and 2C).

\section{Tanshinone IIA enhanced Nrf2 activation and alleviated renal oxidative stress}

Renal MDA and 8-OHdg levels increased in rats with ioversol treatment. With Tanshinone IIA pretreatment, renal MDA and 8-OHdg were alleviated (Fig. 3A and 3B). To explore the mechanism of anti-oxidation, we evaluated Nrf2 activation in the nucleus and found it was enhanced in Tan group (Fig. 3C). Moreover, renal HO-1 expression, the downstream gene of Nrf2, was up-regulated in the Tan group compared to the Veh and Iov groups (Fig. 3D).

\section{Tanshinone IIA reduced oxidative stress and enhanced Nrf2 activation in vitro}

An oxidative injury model with HK2 cells was used to investigate the influence of Tanshinone IIA on Nrf2, (Fig. 4). ROS levels increased 24h following $\mathrm{H}_{2} \mathrm{O}_{2}$ treatment, while Tanshinone IIA reduced ROS levels (Fig. 4A). Moreover, Tanshinone IIA increased activated 
Nrf2 expression and ARE activity as well as upregulated HO-1 (Fig. 4B, 4D and 4E). Additionally, $\mathrm{HO}-1$ activity in $\mathrm{H}_{2} \mathrm{O}_{2}$ treated HK2 cells was elevated, but there was no significant difference between the $\mathrm{H}_{2} \mathrm{O}_{2}$ group and Tanshinone IIA group (Fig. 4C).

\section{Discussion}

In the present study, we observed that Tanshinone IIA pretreatment protected against renal tubular injury and improved renal function in a rat CIN model. Meanwhile, cell apoptosis, uKIM-1 and sNGAL levels were reduced. Tanshinone IIA decreased oxidative stress in injured kidney tissues and HK2 cells under oxidative condition. Nrf2/ARE enhancement and HO-1 up-regulation were observed with Tanshinone IIA pretreatment both in vivo and in vitro. However, Tanshinone IIA did not change HO-1 activity in vitro.

CIN is a very common iatrogenic acute kidney injury [2226]. There are many circumstances in which it is medically necessary to utilize contrast-enhanced imaging, despite the significant risk of CIN. Oxidative stress and inflammation play an important role in the pathogenesis of many kidney injuries including CIN [27-30]. Contrast media induces ROS that causes lipid peroxidation and inflammation. Development or identification of new drugs that can scavenge ROS has been a major focus for the prevention of CIN in high-risk patients [31].

In traditional Chinese medicine, natural herbs and plants are used to treat numerous diseases. Many molecules extracted from plants have therapeutic value and are commonly used in the treatment of human diseases. For instance, artemisinin from Artemisia annua $L$. is widely used to treat malaria [32]. Tanshinone IIA is a key active monomer extracted from

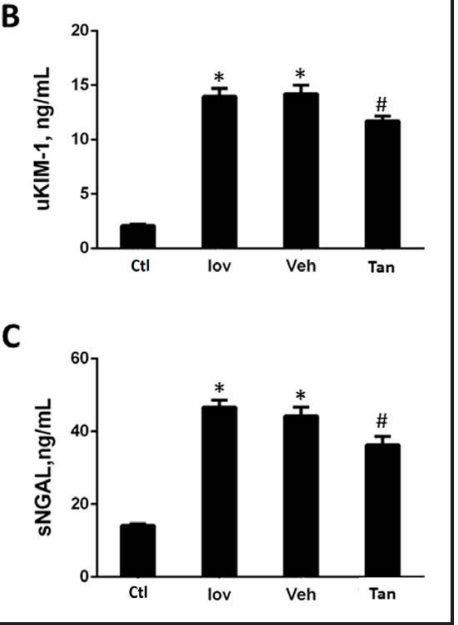

Fig. 2. Tanshinone IIA reduced renal apoptosis, KIM-1 and NGAL A, TUNEL staining for kidney tissues(400X); B, urinary KIM-1; C, serum NGAL $* P<$ 0.05 , versus Ctl group. \#P<0.05, versus Veh group.

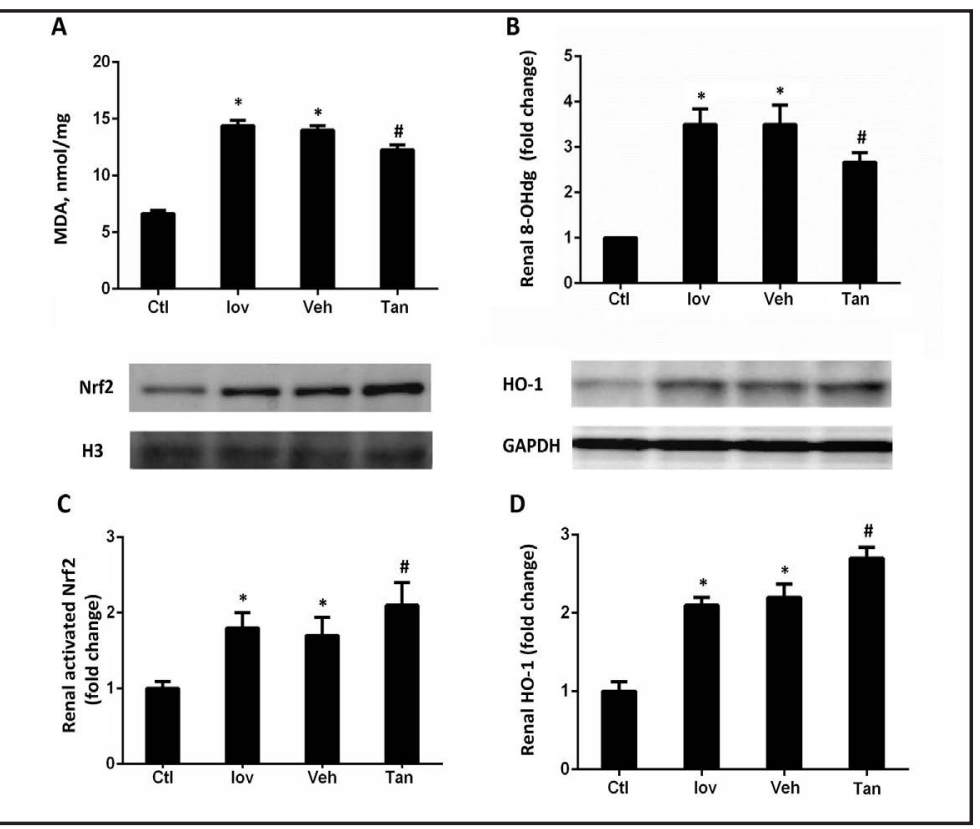

Fig. 3. Tanshinone IIA alleviated oxidative stress via activating Nrf2 in vivo A, renal MDA; B, renal 8-OHdg; C, renal Nrf2 activation; D, renal HO-1 expression $* P<0.05$, versus Ctl group. $\# P<0.01$, versus Veh group. 
Fig. 4. Tanshinone IIA decreased oxidative stess via Nrf2 in vitro A, ROS level; $\mathrm{B}, \mathrm{ARE}$ activation; C, HO-1 activity assay; D, nuclear Nrf2 activiation; E, HO-1 expression $* P<0.05$, versus Ctl group. $\# P<0.05$, versus Veh group.
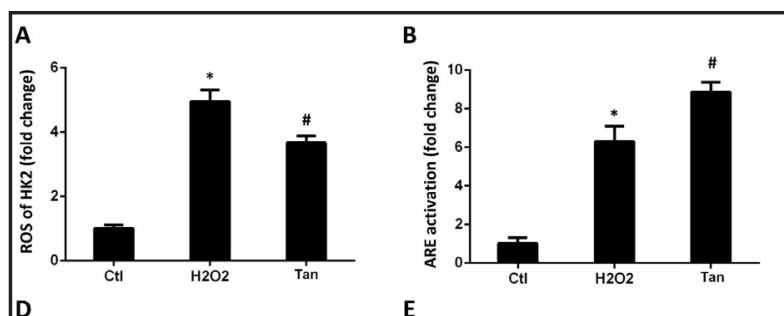

C

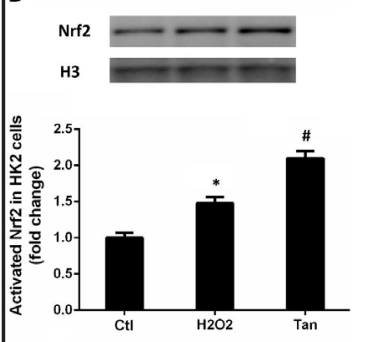

E
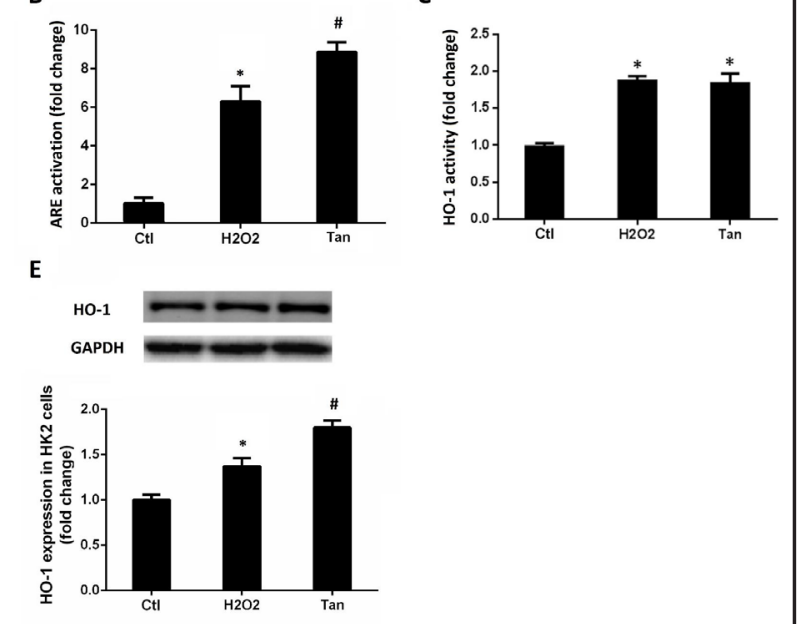

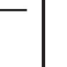

S. miltiorrhiza [8] and has been used to treat cardiovascular and cerebrovascular diseases. In this study, Tanshinone IIA preconditioned rats with CIN exhibited lower levels of oxidative stress. The results were consistent with previous studies which focused on Tanshinone IIA for neurologic injury [15]. In the present study, we demonstrated that Tanshinone IIA has protective effects on CIN, and that these protective effects are related, at least in part, to its anti-oxidative properties.

The anti-oxidative molecular mechanism of Tanshinone IIA is still unclear. A recent study reported that Tanshinone IIA could up-regulate the expression of Bcl-2, an anti-apoptotic protein [10]. On another hand, it was also reported Nrf2 activation contributed to Tanshinone IIA induced neuroprotection by inducing transcription of large numbers of antioxidant genes including heme oxygenase-1 (HO-1) [15]. We hypothesized that Tanshinone IIA might play the same role in preventing CIN. Thus, we performed Nrf2 expression assay and found that Nrf2/ARE pathway was activated by Tanshinone IIA pretreatment both in vivo and in vitro in the present study. Furthermore, HO-1, which is the ARE-regulated antioxidant enzyme, was also up-regulated by Tanshinone IIA. There was no significant difference of HO-1 activity between the $\mathrm{H}_{2} \mathrm{O}_{2}$ group and Tanshinone IIA pretreatment group in vitro, suggesting that the anti-oxidative effects of Tanshinone IIA only depend on Nrf2 induced HO-1 upregulation, but not on HO-1 activity change.

Under non-stressful conditions, Nrf2 is maintained at low levels due to rapid degradation via Keap1-dependent ubiquitin conjugation [33,34]. When stress increases, Nrf2 is activated and exerts anti-oxidative effects. We deduced that Tanshinone IIA pretreatment could enhance Nrf2/ARE activation and play a renoprotective role. Another mechanism for Nrf2 activation may be through the MAP kinase or PI3-k/Akt pathways [35]. A previous study suggested that in human aortic smooth muscle cells, Tanshinone IIA-induced Nrf2 activation was associated with activation of ERK and PKB [36]. Further investigation is needed to elucidate the detailed molecular mechanisms involved in the protective effects of Tanshinone IIA on CIN.

\section{Conclusion}

This study suggested that Tanshinone IIA exhibits renal protection in CIN models through antioxidative and antiapoptotic pathways. Nrf2/ARE activation might be one of the underlying mechanisms of Tanshinone IIA. Tanshinone IIA could be a novel antioxidant to CIN therapy in the future. 


\section{Cellular Physiology Cell Physiol Biochem 2018;46:2616-2623

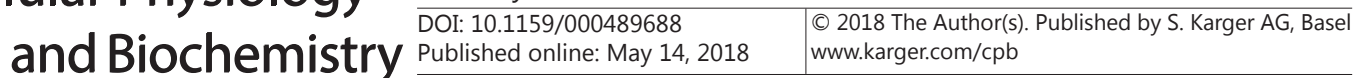

Liang et al.: Tanshinone Protects Against CIN

\section{Acknowledgements}

This work was sponsored by the National Natural Science Foundation of China (81570603 and 81770741) and Health and Family Planning Commission of Shanghai Xuhui District (SHXH201605) and Jiangsu Province Foundation (Z201609). Dr. Guangyuan Zhang is now working in the Department of Urology, affiliated Zhongda Hospital of Southeast University.

\section{Disclosure Statement}

No conflict of interests exists.

\section{References}

1 Wang F, Li J, Huang B, Zhao Q, Yu G, Xuan C, Wei M, Wang N: Clinical survey on contrast-induced nephropathy after coronary angiography. Ren Fail 2013;35:1255-1259.

2 Lu Z, Cheng D, Yin J, Wu R, Zhang G, Zhao Q, Wang N, Wang F, Liang M: Antithrombin III Protects Against Contrast-Induced Nephropathy. Ebiomedicine 2017;17:101-107.

-3 Tan X, Zheng X, Huang Z, Lin J, Xie C, Lin Y: Involvement of s100a8/a9-tlr4-nlrp3 inflammasome pathway in contrast-induced acute kidney injury. Cell Physiol Biochem 2017;43:209-222.

-4 James MT, Samuel SM, Manning MA, Tonelli M, Ghali WA, Faris P, Knudtson ML, Pannu N, Hemmelgarn BR: Contrast-induced acute kidney injury and risk of adverse clinical outcomes after coronary angiography: A systematic review and meta-analysis. Circ Cardiovasc Interv 2013;6:37-43.

-5 Zhao Q Yin J, Lu Z, Kong Y, Zhang G, Zhao B, Wang F: Sulodexide protects contrast-induced nephropathy in sprague-dawley rats. Cell Physiol Biochem. 2016;40:621-632.

6 Zhang Y, Zhang B, Xu DQ Li WP, Xu M, Li JH, Xie XY, Fan QX, Liu W, Mu DG, Dong HY, Wang YX, Nan YD, Li ZC, Jin FG: Tanshinone iia attenuates seawater aspiration-induced lung injury by inhibiting macrophage migration inhibitory factor. Biol Pharm Bull 2011;34:1052-1057.

7 Heyman SN, Rosen S, Khamaisi M, Idee JM, Rosenberger C: Reactive oxygen species and the pathogenesis of radiocontrast-induced nephropathy. Invest Radiol 2010;45:188-195.

$>8$ Chen Y, Wu X, Yu S, Fauzee NJ, Wu J, Li L, Zhao J, Zhao Y: Neuroprotective capabilities of tanshinone iia against cerebral ischemia/reperfusion injury via anti-apoptotic pathway in rats. Biol Pharm Bull 2012;35:164-170.

9 Wen PY, Li J, Lu BL, Liu J, Yang FZ, Zhou L, Luo H, Li WW, Zhou J: Tanshinone iia increases levels of neun, protein disulfide isomerase, and na+/k+-atpase and decreases evidence of microglial activation after cerebral ischemic injury. Neuroreport 2016;27:435-444.

10 Xu YM, Ding GH, Huang J, Xiong Y: Tanshinone iia pretreatment attenuates ischemia/reperfusion-induced renal injury. Exp Ther Med 2016;12:2741-2746.

-11 Tang Q, Han R, Xiao H, Shen J, Luo Q, Li J: Neuroprotective effects of tanshinone iia and/or tetramethylpyrazine in cerebral ischemic injury in vivo and in vitro. Brain Res 2012;1488:81-91.

12 Park EJ, Zhao YZ, Kim YC, Sohn DH: Preventive effects of a purified extract isolated from salvia miltiorrhiza enriched with tanshinone i, tanshinone iia and cryptotanshinone on hepatocyte injury in vitro and in vivo. Food Chem Toxicol 2009; 47:2742-2748.

13 Zhao X, Sun G, Zhang J, Ting SM, Gonzales N, Aronowski J: Dimethyl Fumarate Protects Brain From Damage Produced by Intracerebral Hemorrhage by Mechanism Involving Nrf2. Stroke 2015;46:1923-1928.

14 Wang C, Li C, Peng H, Ye Z, Zhang J, Liu X, Lou T: Activation of the nrf2-are pathway attenuates hyperglycemia-mediated injuries in mouse podocytes. Cell Physiol Biochem 2014;34:891-902.

15 Cai M, Guo Y, Wang S, Wei H, Sun S, Zhao G, Dong H: Tanshinone iia elicits neuroprotective effect through activating the nuclear factor erythroid 2-related factor-dependent antioxidant response. Rejuvenation Res 2017;20:286-297.

16 Wang F, Zhang G, Zhou Y, Gui D, Li J, Xing T, Wang N: Magnolin protects against contrast-induced nephropathy in rats via antioxidation and antiapoptosis. Oxid Med Cell Longev 2014;2014:203458. 


\section{Cellular Physiology Cell Physiol Biochem 2018;46:2616-2623 \begin{tabular}{l|l} 
DOI: 10.1159/000489688 & $\begin{array}{l}\text { O 2018 The Author(s). Published by S. Karger AG, Basel } \\
\text { www.karger.com/cpb }\end{array}$ \\
\hline
\end{tabular}}

Liang et al.: Tanshinone Protects Against CIN

17 Melnikov VY, Faubel S, Siegmund B, Lucia MS, Ljubanovic D, Edelstein CL: Neutrophil-independent mechanisms of caspase-1-and il-18-mediated ischemic acute tubular necrosis in mice. J Clin Invest 2002;110:1083-1091.

18 Yang F, Huang X, Yi T, Yen Y, Moore DD, Huang W: Spontaneous development of liver tumors in the absence of the bile acid receptor farnesoid x receptor. Cancer Res 2007;67:863-867.

19 Yang R, Le G, Li A, Zheng J, Shi Y: Effect of antioxidant capacity on blood lipid metabolism and lipoprotein lipase activity of rats fed a high-fat diet. Nutrition 2006;22:1185-1191.

20 Yang Q, Wang Y, Yang Q, Gao Y, Duan X, Fu Q, Chu C, Pan X, Cui X, Sun Y: Cuprous oxide nanoparticles trigger er stress-induced apoptosis by regulating copper trafficking and overcoming resistance to sunitinib therapy in renal cancer. Biomaterials 2017;146:72-85.

21 Bolignano D, Donato V, Coppolino G, Campo S, Buemi A, Lacquaniti A, Buemi M: Neutrophil gelatinaseassociated lipocalin (ngal) as a marker of kidney damage. Am J Kidney Dis 2008;52:595-605.

22 Lu Z, Yin J, Zhang G, Wu R, Zhao Q, Wang N, Yan C, Wang F: Underestimated incidence of kidney disease in nonrenal outpatient. Ren Fail 2017;39:328-332.

-23 Wu R, Kong Y, Yin J, Liang R, Lu Z, Wang N, Zhao Q, Zhou Y, Yan C, Wang F, Liang R: Antithrombin III is a Novel Predictor for Contrast Induced Nephropathy After Coronary Angiography. Kidney Blood Press Res 2018;43:170-180.

24 Lu Z, Liu N, Wang F: Epigenetic Regulations in Diabetic Nephropathy. J Diabetes Res 2017;2017:7805058.

25 Yin J, Wang F, Kong Y, Wu R, Zhang G, Wang N, Wang L, Lu Z, Liang M: Antithrombin III prevents progression of chronic kidney disease following experimental ischaemic-reperfusion injury. J Cell Mol Med 2017;21:3506-3514.

-26 Kong Y, Yin J, Cheng D, Lu Z, Wang N, Wang F, Liang M: Antithrombin III Attenuates AKI Following Acute Severe Pancreatitis. Shock 2017;DOI: 10.1097/SHK.0000000000000946.

-27 Chen X, Wu R, Kong Y, Yang Y, Gao Y, Sun D, Liu Q, Dai D, Lu Z, Wang N, Ge S, Wang F: Tanshinone IIA attenuates renal damage in STZ-induced diabetic rats via inhibiting oxidative stress and inflammation. Oncotarget 2017;8:31915-31922.

28 Lu Z, Wang F, Liang M: SerpinC1/Antithrombin III in kidney related diseases. Clin Sci (Lond) 2017;131:823831.

29 Yao Y, Chen S, Cao M, Fan X, Yang T, Huang Y, Song X, Li Y, Ye L, Shen N, Shi Y, Li X, Wang F, Qian Y: Antigenspecific CD8+ T cell feedback activates NLRP3 inflammasome in antigen-presenting cells through perforin. Nat Commun 2017; 8:15402.

30 Wu R, Liu X, Yin J, Wu H, Cai X, Wang N, Qian Y, Wang F: IL-6 receptor blockade ameliorates diabetic nephropathy via inhibiting inflammasome in mice. Metabolism 2018; 83:18-24.

-31 Mohammed NM, Mahfouz A, Achkar K, Rafie IM, Hajar R: Contrast-induced nephropathy. Heart Views 2013;14:106-116.

-32 Tu Y: The discovery of artemisinin (qinghaosu) and gifts from chinese medicine. Nat Med 2011;17:12171220.

-33 Kwak M-K, Wakabayashi N, Greenlaw JL, Yamamoto M, Kensler TW: Antioxidants enhance mammalian proteasome expression through the keap1-nrf2 signaling pathway. Mol Cell Biol 2003;23:8786-8794.

-34 McMahon M, Itoh K, Yamamoto M, Hayes JD: Keap1-dependent proteasomal degradation of transcription factor nrf2 contributes to the negative regulation of antioxidant response element-driven gene expression. J Biol Chem 2003;278:21592-21600.

-35 Dai G, Vaughn S, Zhang Y, Wang ET, Garcia-Cardena G, Gimbrone MA, Jr.: Biomechanical forces in atherosclerosis-resistant vascular regions regulate endothelial redox balance via phosphoinositol 3-kinase/ akt-dependent activation of nrf2 Circ Res 2007;101:723-733.

-36 Zhang HS, Wang SQ: Nrf2 is involved in the effect of tanshinone iia on intracellular redox status in human aortic smooth muscle cells. Biochem Pharmacol 2007;73:1358-1366. 